\title{
Insulinas análogas: responsabilidade do SUS e a judicialização.
}

Analog Insulins: SUS liability and the judicialization

Las insulinas similares: la responsabilidad del SUS y la judicialización

\author{
Jorge Alberto Lima ${ }^{1}$ \\ Ana Francisca Kolling ${ }^{2}$ \\ Alexandre Pinto De Paiva Nasser ${ }^{3}$ \\ Antônio Ferreira Marques ${ }^{4}$ \\ Rogger Diquiqui ${ }^{5}$
}

Resumo: Trata-se de um estudo descritivo, epidemiológico e analítico com abordagem qualiquantitativa. $\mathrm{O}$ artigo buscou identificar a relação entre cobertura da Estratégia de Saúde da Família e o aumento de ações judiciais de insulinas análogas nas regiões Sul e Nordeste do Brasil. Nos anos 2010, 2011 e 2012, o consumo de insulinas análogas foi crescente tanto na região sul quanto nordeste. A região sul, apesar de ter um SUS organizado, IDH desenvolvido e educação estruturada, apresentou falhas no programa de diabetes. Em contrapartida, a região nordeste possui IDH baixo na maioria dos municípios e educação deficiente, porém, observou-se a necessidade de reorganização dos programas voltados ao atendimento de usuários portadores de doenças crônicas, em especial, a diabetes, nessa região. Entende-se, que o fortalecimento da atenção básica como porta de entrada para o SUS, nas regiões estudadas pode minimizar o problema da judicialização da saúde.

Palavras-chave: Judicialização, Sistema Único de Saúde, Insulinas Análogas, Estratégia de Saúde da Família, Ações Judiciais.

Abstract: This is a descriptive, epidemiological and analytical approach study, within a qualitative-quantitative perspective. The article sought to identify the relationship between coverage of the Family Health Strategy and the increase in lawsuits related to analog insulin in the South and Northeast regions of Brazil. In the years 2010, 2011 and 2012, analog insulins consumption grew over the years in the South as in the Northeast. The Southern region, despite having an organized SUS, high HDI and a wellstructured education, had flaws in the national diabetes program. In contrast, the Northeast region has a low HDI in most municipalities and poor education; however, there is a need for the reorganization of programs aimed at users with chronic diseases, especially diabetes in this region. It is

\footnotetext{
${ }^{1}$ Jorge Alberto Lima - Mestre em Saúde Coletiva - Universidade de Brasília, Odontólogo Ministério da Saúde, Departamento de Logística, Coordenação de Demanda Judicial, Brasília - DF - Brasil. E-mail: jorge.lima@saude.gov.br.

${ }^{2}$ Ana Francisca Kolling - Mestre em Epidemiologia - Universidade Federal do Rio Grande do Sul, Enfermeira, Departamento de Logística, Coordenação Geral de Análise da Contratação de Insumos Estratégicos, Brasília - DF, Brasil. E-mail: ana.kolling@saude.gov.br.

${ }^{3}$ Alexandre Pinto de Paiva Nasser - Consultor Farmacêutico Ministério da Saúde, Departamento de

Logística, Coordenação de Demanda Judicial. E-mail: alexandre.nasser@saude.gov.br

4 Antônio Ferreira Marques - Consultor, Economista Fiotec, Departamento de Logística, Divisão de Contratações Diretas, Brasília-DF, Brasil. E-mail: antonio.marques@saude.gov.br

${ }^{5}$ Rogger Diquiqui - Consultor Farmacêutico Ministério da Saúde, Departamento de Logística, Coordenação de Demanda Judicial. E-mail: rogger.diquiqui@saude.gov.br
} 
understood that the strengthening of primary health care as a gateway to the Unified Health System in the studied regions can minimize the problem of judicialization in healthcare.

Keywords: Judicialization, Healthcare System, Analog Insulins, the Family Health Strategy, Lawsuits.

Resumen: Se trata de un estudio descriptivo, epidemiológico y analítico con enfoque cualicuantitativa. El trabajo tiene como objetivo identificar la relación entre la estrategia de salud para la cobertura de la familia y el aumento de las demandas de las insulinas análogas en el sur y noreste de Brasil. En los años 2010, 2011 y 2012, el consumo de los análogos ha ido creciendo tanto en el sur como al noreste. La región del sur, a pesar de tener un sistema de salud organizad, Indice de Desarrollo Humano (IDH) desarrollado y estructurado de educación, presentó fallas en el programa de la diabetes. Por el contrario, la región noreste tiene un bajo IDH en la mayoría de los municipios y la educación deficiente. Sin embargo, no había la necesidad de reorganización de los programas destinados a los portadores de enfermedades crónicas, especialmente diabetes en esta región. Se entiende que el fortalecimiento de la atención primaria como puerta de entrada para el sistema público de salud, las regiones pueden minimizar el problema de la judicialización de la salud.

Palabras-Ilave: Judicialización, Sistema de Salud, insulinas analógicas, la Estrategia Salud de la Familia. Decisiones judiciales.

\section{INTRODUÇÃO}

O Sistema Único de Saúde (SUS), inscrito na Constituição Federal de 1988, traz em seus artigos 196 a 200, princípios e diretrizes que o caracteriza como o maior sistema de saúde pública do mundo. O grande desafio reside na gestão compartilhada pela descentralização e autonomia em cada esfera de governo, aliado ao reduzido orçamento para o desenvolvimento de ações e serviços de saúde. Exemplo disso acontece no tratamento de doenças crônicas como diabetes, em que o usuário é inserido em um programa que contempla ações transversais das três esferas do governo.

No Brasil, mesmo com a existência do SUS, gratuito e universal, o custo individual de uma doença crônica é bastante alto, em função dos custos agregados, o que contribui para o empobrecimento das famílias. A forma de responder socialmente, através dos sistemas de atenção à saúde, às condições agudas e crônicas, é que marca as diferenças entre a atenção à demanda espontânea e a atenção programada (1).

Para o acesso aos medicamentos antiglicêmicos, os usuários são cadastrados pelos serviços de saúde dos municípios. O processo se dá pela Estratégia de Saúde da Família que recebe os medicamentos dos Estados, adquiridos por licitação pela União. No entanto, problemas estruturais, desde a questão orçamentária até o planejamento das ações e 
serviços faz com que ocorra eventual desabastecimento dos medicamentos previstos nos Programas de Controle do Diabetes e o paciente recorra à justiça.

Todos esses desencontros provocados ou pela falta da oferta do medicamento, ou pelo desejo de medicamento não contemplado nos programas oficiais, provocam no usuário, o desejo de buscar o seu direito à saúde, constituindo o fenômeno que se convencionou chamar de 'judicialização da saúde'. O Sistema Único de Saúde vem sendo normatizado e implementado, ao longo dos últimos 25 anos, pautado pela universalidade, integralidade, equidade e isto não tem sido, uma tarefa fácil.

No Brasil, mesmo com a existência do Sistema Único de Saúde, gratuito e universal, o custo individual de uma doença crônica é bastante alto, em função dos custos agregados, o que contribui para o empobrecimento das famílias (...) a forma de responder socialmente, através dos sistemas de atenção à saúde, às condições agudas e crônicas, é que marca as diferenças (2).

De acordo com o Ministério da Saúde e Organização Pan-Americana da Saúde, custos diretos para o atendimento ao diabetes, variam, mundialmente, de $2,5 \%$ a $15 \%$ dos gastos nacionais em saúde, dependendo da prevalência local de diabetes e da complexidade do tratamento disponível (3).

Apesar dos avanços conquistados por meio de uma legislação arrojada, constata-se que os usuários de saúde com diabetes, ainda hoje, têm pouco acesso a esses benefícios, exercendo minimamente seus direitos como cidadãos (4).

O medicamento glibenclamida é o medicamento mais utilizado para tratar a diabetes. Reconhece-se que houve avanço no que diz respeito à criação de leis e normas que assegurem o acesso do usuário aos serviços de saúde, mas, por outro lado, a operacionalização das leis esbarra na falta de mecanismos que sensibilizem os gestores e profissionais de saúde em relação às suas responsabilidades e conscientizem a população sobre os direitos que lhe são assegurados por lei (5).

Análise, ainda que sucinta, das principais políticas e programas demonstra que na atenção básica, a Estratégia Saúde da Família tem encontrado dificuldades para sua expansão nos municípios de maior porte e continua com problemas nas áreas de gestão, de recursos humanos e de articulação com o restante da rede de serviços (6). 


\section{Sobre Judicialização da Saúde}

Muito se tem publicado a respeito da chamada judicialização da saúde, no Brasil e internacionalmente, o crescente número de ações judiciais propostas por usuários do Sistema Único de Saúde para garantir o fornecimento de medicamentos tem sido motivo de preocupações para os gestores em todos os níveis federativos (7) (8). A questão é complexa não somente porque envolve diversos atores-gestores do SUS, magistrados, profissionais de saúde e cidadãos, mas, porque o tema medicamentos não está relacionado apenas a decisões sobre políticas de saúde, como também, a decisões técnicas à incorporação de tecnologia. Nesse sentido, o Judiciário não só estaria intervindo na política de saúde, mas também decidindo sem o necessário conhecimento técnico (7).

A maioria dos dados sobre a judicialização da saúde refere-se às ações judiciais e gastos do Ministério da Saúde e dos Estados da federação. Salvo raras exceções (9), pouco se tem pesquisado sobre o impacto deste fenômeno nos municípios. Estudar o peso das ações judiciais com pedido de medicamentos nos orçamentos municipais é de grande importância uma vez que os municípios, em geral, possuem um orçamento menor do que os estados e a União, o que significa que os efeitos desse processo são ainda mais relevantes em termos de comprometimento do orçamento público e, portanto, dos recursos necessários para a execução de outras políticas públicas no setor da saúde. Esta condição determina, a priori, uma menor capacidade municipal em lidar com a imprevisibilidade advinda dos gastos em saúde ordenados judicialmente, uma vez que os recursos totais disponíveis para remanejamento ao cumprimento destas decisões também tendem a ser de menor monta.

No tocante à execução da assistência farmacêutica na atenção básica, a aquisição poderá tanto ser feita pelo estado, que repassará os medicamentos em espécie para os municípios, como diretamente pelos municípios, com seus recursos e os recebidos do Ministério da Saúde e do estado. Além disso, o Ministério da Saúde é responsável pela aquisição e distribuição das Insulinas Humanas NPH e Regular (...) que depois são distribuídos a Estados e Municípios (9).

A importância da problemática de que os entes federados procure por meio de novas pactuações através da Comissão Intergestores Bipartite $(C I B)$ e depois na Comissão Intergestores Tripartite (CIT), buscando uma melhor solução de ofertar dos medicamentos para diabetes, inclusive as insulinas análogas, requer um esforço grande, porque existe o 
entrave orçamentário como dificultador da proposta de melhorar o modelo de atenção à saúde por parte dos gestores.

\section{Metodologia}

Tratou-se de um estudo epidemiológico descritivo e analítico com abordagem qualiquantitativa de base documental e jurisprudencial. A unidade de análise foram as decisões judiciais contra os entes federados em que conste insulinas análogas ou insulinas $\mathrm{NPH}$ ou regular como objeto material do pedido do autor. A base de dados da pesquisa foi o SISCAWEB/MS, ou seja, Sistema de Ação Judicial do Ministério da Saúde, no período de 2010 a 2012, nos estados das regiões nordeste e sul.

As regiões foram selecionadas pelo número de ações tuteladas pelos entes municipais de vários estados dessas regiões e disponíveis na Coordenação de Compra por Determinação Judicial do Ministério da Saúde (CDJU/MS).

Coletados os dados, estes foram inseridos em matrizes e trabalhados para gerar resultados, dispostos em gráficos.

A execução da pesquisa não envolveu qualquer tipo de intervenção em seres humanos, ainda assim, o projeto foi submetido ao Comitê de Ética em Pesquisa da Universidade de Brasília por meio da Plataforma Brasil, com pedido de dispensa, por se tratar da transcrição de dados secundários.

O artigo ainda utilizou dados do Instituto Brasileiro de Geografia e Estatística (IBGE) e do índice de Desenvolvimento Humano (IDH) do Atlas de Desenvolvimento Humano.

\section{Resultados}

Os tipos de insulinas análogas encontradas na pesquisa foram: tipo Glargina de $3 \mathrm{ml}$ e de $10 \mathrm{ml}$, insulina tipo Aspart de $3 \mathrm{ml}$ e $10 \mathrm{ml}$, insulina Detemir de 3ml, insulina Lispro de $3 \mathrm{ml}$ e de $10 \mathrm{ml}$ e insulina Glulisina $3 \mathrm{ml}$, além de comprimidos de Metformina e glibenclamida utilizados para diabetes.

Conforme as figuras 1 e 2, observamos na região sul os homens foram os maiores consumidores nos três anos da pesquisa, sendo 2010 (68\%), 2011 (67\%) e 2012 (65\%), e que apenas em 2012 a proporção homem/mulher foi de 13 mulheres para 12 homens. 
Figura 1 - Consumo Anual de Insulina conforme Sexo na Região Sul

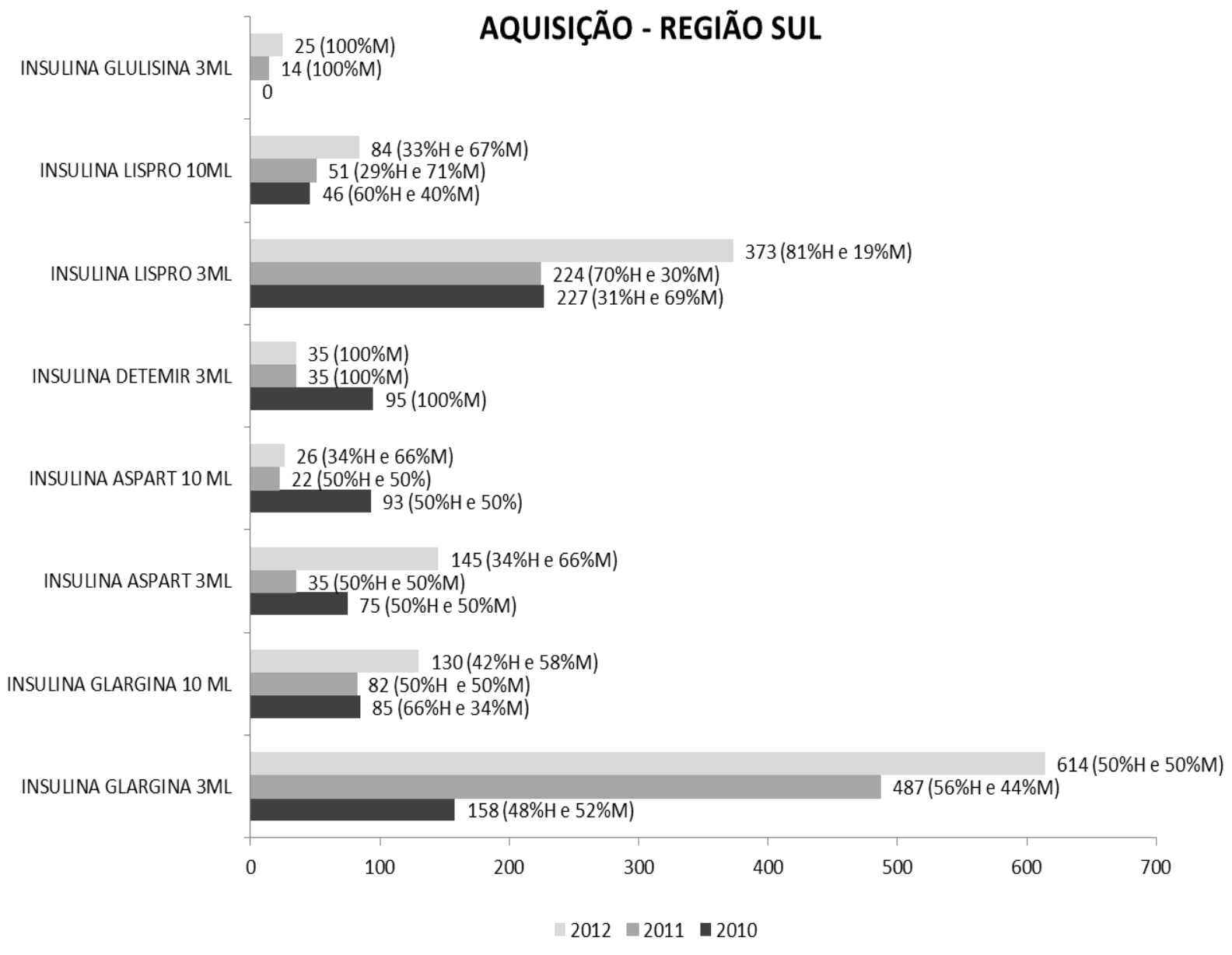

Fonte: Sistema de Informação-SISCAWEB/CGIES/CDJU/2014.

No nordeste, os homens também foram os maiores consumidores dessas insulinas nos três anos da pesquisa e que em 2012 foi o maior ano do consumo (64\%), e apesar das mulheres serem maiores demandantes, o consumo foi menor. 
Figura 2 - Consumo Anual de Insulina conforme sexo na Região Nordeste

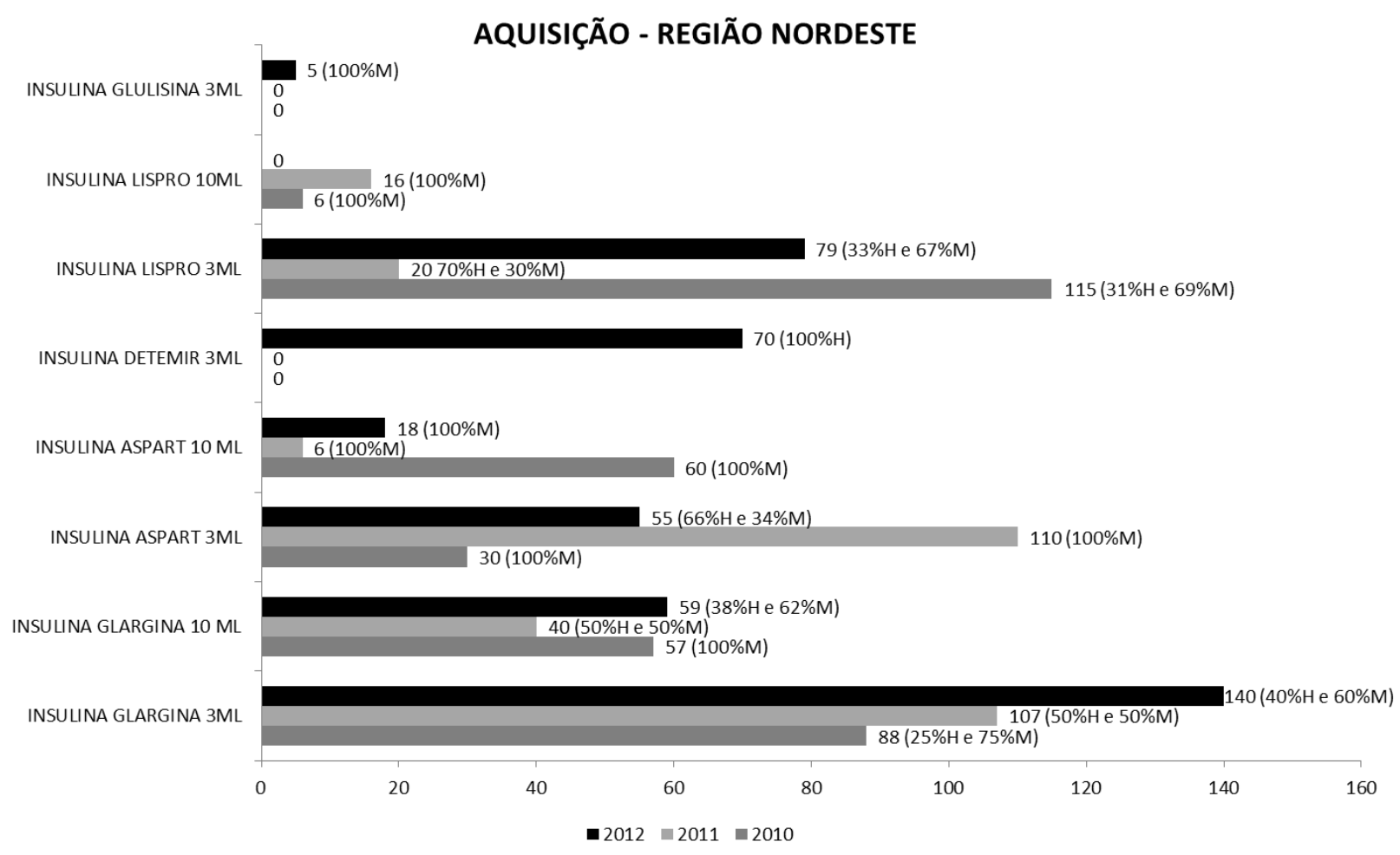

Fonte: Sistema de Informação-SISCAWEB/CGIES/CDJU/2014.

Em um estudo sobre a qualidade de vida de pacientes com hipertensão e diabetes por uma equipe de saúde da família no Brasil em 2008, caracterizou o sexo feminino com predominância de $66,7 \%$, pois no Planeta, a população feminina é maior que a masculina, segundo dados mundiais. Este fato explica, em parte, a maior proporção de mulheres acometidas, e ainda que sejam diagnosticadas por procurarem mais frequentemente os serviços de saúde. Alguns estudos têm demonstrado que a hipertensão e diabetes mellitus tem maior prevalência em indivíduos acima de 35 anos, e com baixa escolaridade (10).

O que pode explicar a razão de os homens serem os maiores consumidores deste tipo de insulina em relação às mulheres foi o tempo expedido pela justiça que pode ter sido de 6 em 6 meses ou de 8 em 8 meses e a quantidade prescrita pelos médicos para a dosagem prescrita.

$\mathrm{Na}$ região sul, as mulheres foram as maiores consumidoras de insulinas glargina de $10 \mathrm{ml}$, e em número maior do que os homens, nos anos de 2010 (7 mulheres e 52\% de 
consumo) e 2012 (5 mulheres e 74\% de consumo). Em 2011, o consumo foi maior entre os homens, porém a proporção homem/mulher foi igual 3.

Na região nordeste, no ano de 2010 não houve nenhum homem consumidor. O consumo foi maior entre as mulheres nos três anos da pesquisa, no mesmo raciocínio do tempo expedido pela justiça que pode ter sido de 6 em 6 meses ou de 8 em 8 meses e a quantidade prescrita pelos médicos para a dosagem prescrita $A$ frequência de adultos que referiram diagnóstico médico prévio de diabetes variou.

No sexo masculino, as maiores frequências foram observadas em Curitiba (8,4\%), e Fortaleza (7,4\%). Entre as mulheres, o diagnóstico de diabetes foi mais frequente em Natal $(9,0 \%)$ e Porto Alegre (8,7\%) e menos frequente em Teresina (4,6\%). No conjunto da população adulta das 27 cidades estudadas, a frequência do diagnostico medico prévio de diabetes foi de 7,4\%, sendo de 6,5\% entre homens e de $8,1 \%$ entre mulheres. Em ambos os sexos, o diagnóstico da doença se tornou mais comum com o avanço da idade, com maior aumento a partir dos 35 anos.

Mais de um quinto dos homens e das mulheres com 65 anos ou mais de idade referiram diagnóstico médico de diabetes. Em ambos os sexos, frequência máxima de diabetes foi encontrada em indivíduos com até oito anos de escolaridade: 10,3\% em homens e $13,7 \%$ em mulheres.

Na região sul não houve nenhum homem consumidor em 2010, sendo que em 2011, a quantidade prescrita é maior do que a das mulheres para o mesmo número de pessoas (25 unidades para 1 pessoa), em 2012, o maior consumo está entre os homens (59\%) embora seja o mesmo número de mulheres (4 homens e 4 mulheres). Em 2010, o consumo foi $100 \%$ entre as mulheres (3).

Na região nordeste, não houve nenhum homem consumidor em 2010 e 2011. O maior consumo foi em 2012 ( 2 homens e $55 \%$ de consumo). As mulheres foram em maior número nos anos de 2010 e 2011 com consumo de 100\%.

$\mathrm{Na}$ pesquisa VIGITEL/MS (inquérito de vigilância epidemiológica realizada por telefone em 2012, pelo Ministério da Saúde) a frequência de adultos que referiram diagnóstico médico prévio de diabetes variou. Em ambos os sexos, o diagnóstico da doença se tornou mais comum com o avanço da idade, com maior aumento a partir dos 35 anos. Mais de um quinto dos homens e das mulheres com 65 anos ou mais de idade referiram diagnóstico médico de diabetes. Em ambos os sexos, frequência máxima de diabetes foi 
encontrada em indivíduos com até oito anos de escolaridade: 10,3\% em homens e 13,7\% em mulheres (11).

Na região sul, a proporção de homens foi igual nos três anos da pesquisa e em relação a quantidade consumida (1homem/10 unidades). As mulheres foram em maior número em 2010 e maiores consumidoras (92\%).

Na região nordeste, não houve homens consumidores em 2010 e 2012, apenas em 2011 , com $100 \%$ do consumo. As mulheres foram 100\% consumidoras em 2010 e em 2012, e também em maior número que os homens (2).

Observa-se que as mulheres consumiram um número muito grande dessas insulinas tanto na região sul quanto na região nordeste, sugere-se que a quantidade prescrita seja para dois períodos de 8 meses para cada 2 pacientes de cada região.

Na região sul, não houve homens consumidores em nenhum dos anos da pesquisa. Em relação às mulheres em 2010, houve um alto consumo de insulinas Detemir para dois usuários (100\%). Não houve consumo entre as mulheres em 2011. Em 2012, o consumo foi normal para duas mulheres (100\%). Na região nordeste, não houve nem homens e nem mulheres consumidores nos anos 2010 e 2011, apenas em 2012, a proporção de mulheres foi maior em quantidade consumida (95\%) para duas mulheres.

Observa-se que as mulheres foram as maiores consumidoras e a quantidade prescrita para o consumo foi para 1 ano (6/6 meses).

As insulinas Glargina e Detemir são análogas de insulina de ação prolongada, lançadas nos últimos anos como alternativas à insulina humana (NPH). Os resultados apresentados e os vieses metodológicos dos estudos clínicos disponíveis atualmente não permitem afirmar que haja diferença entre as insulinas Glargina, Detemir e NPH, no que se refere ao controle glicêmico. Apesar dos resultados indicarem superioridade das insulinas análogas quanto à redução do risco de hipoglicemia, os problemas identificados nos estudos podem comprometer a validade desses achados (12).

Na região sul, houve um alto consumo desta insulina com destaque para os homens em 2011 (64\%) e 2012 (85\%), em relação às mulheres, e que em 2010, houve um grande consumo e maior número de mulheres (9 mulheres e 71\% consumo). No nordeste, em 2010, não houve homem consumidor e em 2011, o consumo foi de $50 \%$ entre homens e mulheres, o destaque ficou para as mulheres em 2010, com um número alto de mulheres e $100 \%$ de consumo. 
O perfil de homens consumidores na região sul foi maior em 2011 e 2012, com grandes quantidades para consumo e em 2010, as mulheres foram maiores consumidoras com quantidades prescritas de 8/8 meses conforme prescritor e dose. Enquanto na região nordeste, o destaque foi para as mulheres em 2010, com as mesmas características da região sul.

Geralmente o fornecimento de insulina de $3 \mathrm{ml}$, relaciona-se a pacientes que fazem uso mensal de uma dosagem inferior a 1000 unidades internacionais (UI), já que em cada tubete há fornecimento de $300 \mathrm{Ui}$. Como as insulinas, regra geral, possuem prazo de validade de 28 dias após abertura do recipiente, sob refrigeração adequada, os tubetes de $3 \mathrm{ml}$ são mais adequados a pacientes que fazem uso inferior a 1000 Ui mensais como uso de canetas injetoras. O tubete é adequado para uso em canetas injetoras. Forma mais prática de administração.

Na região sul em 2010, houve maior proporção de homens consumidores em relação às mulheres (78\% e 12\%), enquanto que em 2011, as mulheres foram as maiores consumidoras e também a maioria (73\% e 27\%). Já no ano de 2012, as mulheres também superaram os homens (71\% e $29 \%)$.

$\mathrm{Na}$ região nordeste, não houve homens consumidores em nenhum ano. Já as mulheres consumiram 100\% em 2010 e 2011. Não houve consumo em 2012.

O destaque fica para o ano de 2010, para os homens na região sul, com maior número de homens e consumo regular para apenas 6 meses, enquanto os anos de 2011 e 2102 as mulheres da região sul foram a maioria, com consumo também regular.

A insulina glulisina na região sul foi demandada apenas nos anos de 2011 e 2012 e foi consumida por pessoas do sexo masculino. Na região nordeste percebe-se que houve 0 contrário, não houve consumo pelo sexo masculino, apenas o sexo feminino consumiu e no ano de 2011. Pode ter havido falhas no sistema de informação, ou o consumo desta insulina foi temporário.

$\mathrm{Na}$ figura 3, percebemos que o consumo de comprimidos de glibenclamida/metformina, se deu na região sul apenas por pessoas do sexo masculino e no ano de 2010, enquanto que as pessoas do sexo feminino consumiram em 2010 e em 2011. Na região nordeste houve consumo apenas no sexo masculino e nos anos de 2011 e 2012 , pode ter havido falhas no sistema de informação e ou não ter tido demanda pelo sexo feminino. 
Figura 3 - Consumo Anual de Comprimidos de Metformina/Glibenclamida de acordo com Sexo e Região do Usuário

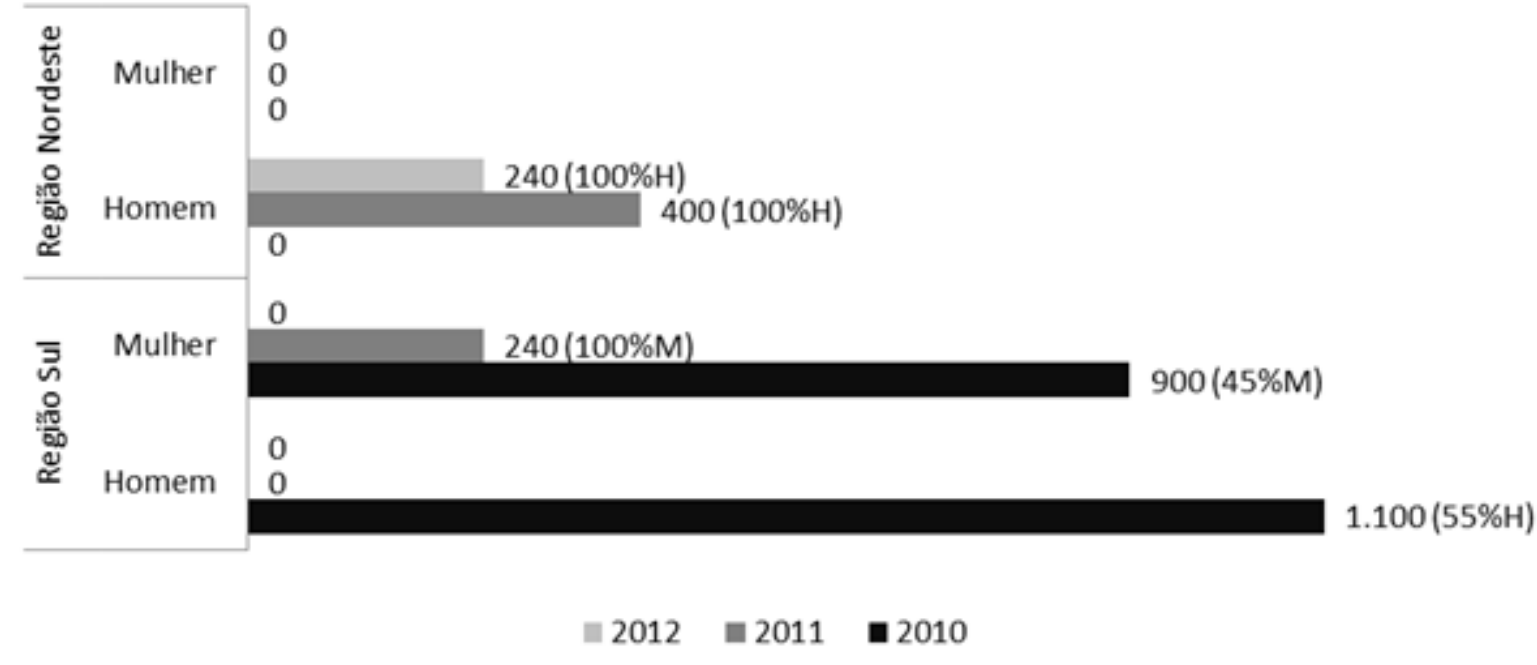

Fonte: Sistema de Informação-SISCAWEB/CGIES/CDJU/2014.

A particularidade é que esses medicamentos fazem parte da relação de medicamentos do SUS e existem nos programas de diabetes dessas duas regiões, o que denota certa fragilidade do controle desses medicamentos em seus estoques.

Nas figuras 4 e 5, observamos as demandas judiciais de todos os tipos de insulina que ocorreram nos anos de 2010, 2011 e 2012 pela Coordenação da Compra por Determinação Judicial, com destaque para os análogos de insulinas Glargina de $3 \mathrm{ml}$ e de $10 \mathrm{ml}$, demandadas nas regiões sul e nordeste e ainda um pouco do percentual de comprimidos de metformina e glibenclamida, que não deveriam estar na demanda pois fazem parte da relação nacional de medicamentos e dos próprios municípios.

A figura 4, apresenta as insulinas aprovadas pelos programas de diabetes do Ministério da Saúde e que são repassadas aos Estados e municípios, e mesmo assim ainda demandaram judicialmente nos anos de 2010 a 2012, isso demonstra fragilidade do Sistema de Saúde Municipal, que precisa corrigir algumas falhas no cadastramento dos usuários que possuem diabetes e manter os estoques em dia. 
Figura 4 - Insulinas consumidas dos programas do Ministério da Saúde

\begin{tabular}{|c|c|c|c|}
\hline \multicolumn{4}{|c|}{ CUSTOS TOTAIS INSUUNAS DOS PROGRAMAS/MS - 2010/2012 - Brasil } \\
\hline Tipo & Quantidade & $\mathrm{N}=$ Paciente & Custo (R\$̦) \\
\hline INSULINA HUMANA NPH3 - 3ML & 329 & 5 & $4.083,45$ \\
\hline INSULINA HUMANA NPH3 - 10ML & 125 & 2 & 67,62 \\
\hline INSULINA HUMANA TIPO REGULAR 3ML & 67 & 4 & 804,00 \\
\hline INSULINA HUMANA TIPO REGULAR 1OML & 206 & 8 & 321,80 \\
\hline TOTAL: & & 19 & $5.276,87$ \\
\hline
\end{tabular}

Fonte: Sistema de Informação - SISCAWEB/CGIES/CDJU/2014.

$\mathrm{Na}$ figura 5, apresentamos todos os tipos de insulinas análogas que foram demandadas por todas as regiões do Brasil nos anos de 2010, 2011 e 2012, o detalhe que se tem em comum nas três figuras acima é que existe um número menor de pacientes e uma quantidade enorme de insulinas sem contar o total de gastos dispendidos pela União. Outro fator que chama a atenção é que se reduzirmos o valor total das insulinas consumidas nas regiões sul e nordeste do valor total da figura 5 notamos que essas duas regiões juntas consumiram quase o total consumido no Brasil.

Figura 5 - Consumo total das insulinas análogas

\begin{tabular}{lrrrr}
\hline \multicolumn{1}{c}{ CUSTOS TOTAIS INSULINAS ANÁLOGAS } & 2010/2011/2012 & - Brasil & \\
\hline \multicolumn{1}{c}{ Tipo } & Quantidade & No Paciente & Custo (R\$̦) \\
INSUUNA ASPART 3ML & 2.830 & 71 & $51.188,69$ \\
INSUUNA ASPART 1OML & 548 & 12 & $16.579,78$ \\
INSUUNA DETEMIR 3M & 1.585 & 22 & $46.875,79$ \\
INSUUNA GLUUSINA 3ML & 596 & 22 & $8.082,87$ \\
INSUUNA GLUUSINA 10ML & 9 & 1 & 449.82 \\
INSUUNA LISPRO 3ML & 2.302 & 54 & $32.874,85$ \\
INSUUNA LISPRO 1OML & 326 & 13 & $9.880,56$ \\
INSUUNA GLARGINA 3ML & 6.222 & 127 & $283.588,57$ \\
INSUUNA GLARGINA 1OML & 1.048 & 53 & $104.704,14$ \\
TOTAL: & & 375 & $553.775,25$ \\
\hline
\end{tabular}

Fonte: Sistema de Informação-SISCAWEB/CGIES/CDJU/2014. 


\section{Discussão}

A preocupação em tratar a diabetes no Brasil indica que os programas de doenças crônicas para tratar a doença nas regiões sul e nordeste, ainda precisam organizar melhor sua estrutura sobre: cadastramento dos usuários até a dispensação dos medicamentos, e controle por parte da equipe da Estratégia de saúde da Família. Contextualizando as regiões sul e nordeste, de acordo com os dados sociodemográficos do censo IBGE 2010 sobre educação populacional, a região sul apresentou melhor índice de desenvolvimento humano e uma educação de boa qualidade, que é confirmada pela preocupação do usuário com sua saúde, por outro lado existe um descaso por parte dos gestores locais quanto à organização dos sistemas de saúde para atingir metas programáticas.

Na região nordeste, o percentual de desenvolvimento humano ainda é preocupante com patamares que vão de baixo e muito baixo, chegando a extrema pobreza, confirmando ainda mais iniquidades de todos os tipos e um nível educacional baixo na maioria da sua população. Existe a preocupação em cuidar da diabetes, porém, esse usuário não possui conhecimento suficiente para o controle da doença, o que também causa preocupação.

Conforme demonstrado na figura 5 , existe preferência pelos análogos de insulina glargina de $3 \mathrm{ml}$ e de $10 \mathrm{ml}$ pelas regiões sul e também pela região nordeste, o que, demonstra que os sistemas de saúde precisam se adequarem melhor, visto que o orçamento da União extrapola, quase todo o valor de insulinas consumidas no Brasil. O crescimento do número de ações judiciais, associado ao fato de que as decisões são predominantemente favoráveis aos autores. Visto que, algumas decisões acabam comprometendo gravemente o orçamento para a saúde, em razão do elevado preço de alguns medicamentos pleiteados, que, em muitos casos, não se encontram nos elencos oficiais da regulamentação da assistência farmacêutica no âmbito do SUS (7).

Outra questão é em relação aos estudos de eficácia comprovados para as insulinas análogas que trazem uma discussão para toda a sociedade, incluindo gestores, formadores de políticas públicas, judiciário e o poder executivo em todas suas instâncias.

A maior parte da literatura científica internacional aponta na direção de que não há evidências fortes de que as insulinas análogas tragam melhoras significativas nas condições de saúde dos pacientes (9) (12). Portanto, existe forte divergência na literatura médicocientífica quanto à maior efetividade (resultados de melhora nas condições de saúde) do tratamento com insulinas análogas. Contudo, uma coisa é certa: as insulinas análogas são 
muito mais custosas que as humanas. Mesmo quando se reconhecem as vantagens das insulinas análogas sobre as humanas, isto não necessariamente significa que sua incorporação valha a pena. Em sentido oposto, outros estudos afirmam que as insulinas análogas possuem melhor relação custo-efetividade em relação às humanas, uma vez que a maior conveniência das primeiras, além de trazer maior comodidade ao paciente, levaria a uma maior adesão ao tratamento (13).

Enquanto se discute sobre a melhor forma de tratar a doença diabetes no Brasil, a mesma continua crescendo em todas as regiões, sendo necessários mais estudos que tragam dados sobre controle da doença utilizando análogos de insulina, e com isso à incorporação nos protocolos clínicos do Ministério da Saúde, o que geraria economia aos cofres públicos e consequente organização das listas de medicamentos oficiais por parte dos entes federados.

\section{Referências}

1 - Mendes EV. O Cuidado das condições crônicas na atenção primária à saúde: o imperativo da consolidação da estratégia da saúde da Família. Brasília: Organização PanAmericana da Saúde, 2012.

2 - Reis AAC et al. A gestão do cuidado na rede de saúde em São Bernardo do Campo: enfrentando desafios e tecendo novos arranjos. In: Sousa MF et al. Saúde da Família nos municípios brasileiros: os reflexos dos 20 anos do espelho do futuro. Campinas, São Paulo: Saberes Editora; 2014. p.206-242.

3 - Pepe VLE et al. A judicialização da Saúde e os novos desafios da Gestão da Assistência Farmacêutica. Ciência e Saúde Coletiva. 2010 [Acesso em 1 abr de 2015] 15(5): 2405-2414. Disponível em

http://www.scielo.br/scielo.php?script=sci arttext\&pid=S1413-81232010000500015

4 - Baptista TWF. O direito à saúde no Brasil: sobre como chegamos no Sistema Único de Saúde e o que esperamos dele. In: Venâncio J. Políticas de saúde. Rio de Janeiro: Editora Fiocruz, 2005.

5 - Lopes LC, Barberato-Filho S, Costa AC, Osorio-de-Castro CGS. Uso racional de medicamentos antineoplásicos e ações judiciais no Estado de São Paulo. Rev. Saúde Pública, 2010, 44 (4):620-628,.

6 - Instituto de Pesquisa Econômica Aplicada - Políticas Sociais: acompanhamento e análise. Brasília: IPEA, 2011. 
7 - Borges DCL, Ugá MAD. As Ações Individuais para o Fornecimento de Medicamentos no Âmbito do SUS: características dos Conflitos e Limites para a Atuação Judicial. Revista de Direito Sanitário. 2009 [Acesso em 25 jan 2015] 10(1): 13-38. Disponível em http://www.revistas.usp.br/rdisan/article/view/13144

8 - Dallari SG. Aspectos particulares da chamada Judicialização da Saúde. Revista de Direito Sanitário. 2013 [Acesso em 25 jan 2015] 14(1):77-81. Disponível em http://www.revistas.usp.br/rdisan/article/view/56624

9 - Wang DWL et al. Os impactos da judicialização da saúde no orçamento Público da Saúde: O caso do Município de São Paulo. In: Anais VI Jornada -Eixo Gestão na Saúde, Financiamento e gasto em saúde. Brasília: Ministério da Saúde; 2012.

10 - Miranzi SSC et al. Qualidade de vida de indivíduos com Diabetes Mellitus e Hipertensão acompanhados por uma Equipe de saúde da Família. In: Miranzi SSC, Ferreira FS, Iwamoto HH, Pereira GA, Miranzi, MAS. Texto Contexto Enfermagem. 2008; 17(4): 672-9.

11 - Ministério da Saúde. VIGITEL Brasil 2012: vigilância de fatores de risco e proteção para doenças crônicas por inquérito telefônico. Brasília: Ministério da Saúde; 2013.

12 - Agência Nacional de Vigilância Sanitária. Insulina Glargina e Insulina Detemir no controle do Diabetes Mellitus tipo I. Boletim Brasileiro de Avaliação de Tecnologias em Saúde (BRATS). Brasília, 2010.

13 - Wang $D$ et al. Judiciário e fornecimento de insulinas análogas pelo sistema público de saúde: Direito, ciência e políticas públicas. Casoteca do Direito GV - Produção de Casos. [Internet] [Acesso em 02 fev 2015] 2011. Disponível em http://direitosp.fgv.br/casoteca. 